\title{
Effect of fortifying breast milk on gastric emptying
}

\author{
R J McClure, S J Newell
}

\begin{abstract}
A study was performed to determine if the addition of a fortifier to expressed breast milk (EBM) affected gastric emptying in low birthweight infants. Using ultrasonography, the gastric emptying of EBM alone was compared with that containing a fortifier, in a blind, crossover study. Twenty two low birthweight infants were studied: median (range) gestation 31.5 weeks (28-37); birthweight $1495 \mathrm{~g}$ (1000$2480 \mathrm{~g}$ ). The gastric antral cross-sectional area (ACSA) was measured by ultrasonography before each feed and then sequentially after its completion until the ACSA returned to its pre-feed value. The half emptying time was calculated as the time taken for the ACSA to decrease to half the maximum increment. The mean difference (standard error) between half emptying times for EBM alone and for EBM with added fortifier was not significant: $1.48(4 \cdot 9)$ minutes.
\end{abstract}

These data show that fortifying breast milk does not affect gastric emptying and suggests that the practice is unlikely to affect feed tolerance in low birthweight infants.

(Arch Dis Child 1996; 74: F60-F62)

Keywords: breast milk, gastric emptying, fortifier, feed tolerance.

In most circumstances feeding low birthweight infants with expressed breast milk (EBM), rather than with preterm formula, is beneficial. EBM provides biologically important non-nutrients such as immunoglobulins, non-antibody mediated immune factors, digestive enzymes, growth factors and hormones, which may promote growth and development as well as having bacteriostatic effects. ${ }^{1-3}$ EBM has the disadvantage of sometimes being nutritionally inadequate for this group of infants. Hypoproteinaemia and inadequacies in calcium, phosphorus, and magnesium supply have been reported. ${ }^{4-6}$ The practice of fortifying EBM is undertaken to improve its nutritional content and has been shown to increase the rate of weight and length gain in low birthweight infants. ${ }^{7-9}$

Several methods are used to fortify EBM, including the addition of volumes of either term or preterm formula. Specifically designed preparations for fortifying are now commercially available and have been rapidly adopted for use by many neonatal units. They are formulated, so that when added to EBM, the resultant milk satisfies the ESPGAN nutritional guidelines for low birthweight infants. ${ }^{10}$
A common problem in low birthweight infants is poor milk tolerance. Gastric emptying is a major determinant of tolerance of milk feeds. Increasing caloric density of milk, ${ }^{11}$ as well as the use of formula milk, ${ }^{12} 13$ are included in the factors known to delay gastric emptying in the preterm infant. The addition of fortifier significantly increases the caloric density of EBM, as well as changing its composition towards that of a formula milk. Therefore, there is a need to test the affect of fortifying breast milk on gastric emptying in low birthweight infants.

Gastric emptying may be assessed using serial ultrasound measurements of the crosssectional area of the gastric antrum. This technique has been successfully validated and used before in preterm infants. ${ }^{14} \mathrm{We}$ used ultrasonic assessment of gastric emptying to compare feeds of EBM alone with that of EBM with added fortifier, in a blind, crossover study.

\section{Methods}

The study was performed on the regional neonatal intensive care unit at St James's University Hospital, Leeds. Infants who satisfied all of the following criteria were recruited into the study: weight of less than $2500 \mathrm{~g}$ at the time of measurement; no evidence of gastrointestinal disease; not receiving drug treatment known to interfere with gastric emptying; and receiving at least $150 \mathrm{ml} / \mathrm{kg} /$ day of $E B M$ by bolus administration, via a nasogastric tube, with an interval of at least two hours between feeds.

Twenty two infants were recruited (13 boys, nine girls). This number was chosen to ensure that the study had at least a $90 \%$ power of revealing a $10 \%$ or greater difference in gastric emptying time. Median (range) birthweight was $1495 \mathrm{~g}$ (1000-2480), gestation 31.5 weeks (28-37), and postnatal age at the time of study 20 (6-67) days. Twelve (55\%) infants were small for gestational age (birthweight below the 10th centile for gestation). All other infants had birthweights appropriate for gestation.

A paired study measuring gastric emptying was performed on each infant, once following a nasogastric feed of EBM alone, and once after a feed of EBM with added fortifier. Fortifier (Breast Milk Fortifier; Cow \& Gate) was added to EBM according to the manufacturer's instructions ( $50 \mathrm{~g}$ added to each $50 \mathrm{ml}$ of breast milk). Each infant was fed the same volume of EBM on each occasion and the two feeds were both thawed from the same batch of frozen stored milk from the infant's own mother. The interval between the two measurements for an individual baby was between 24 and 48 hours and infants were randomly assigned as to which feed to receive 


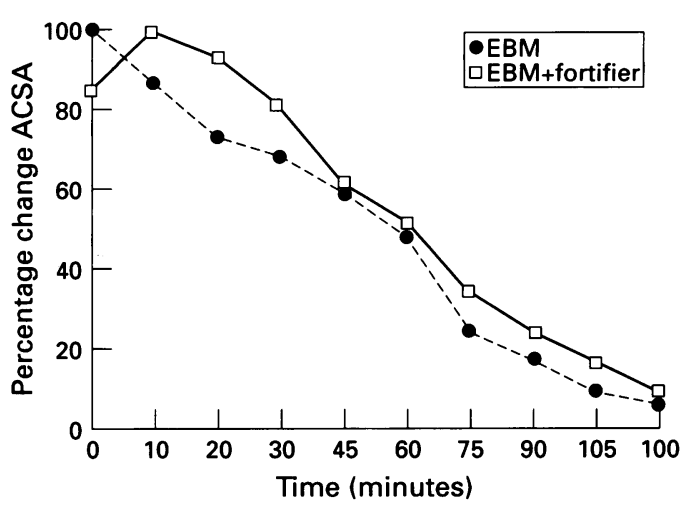

Figure 1 Mean percentage change in ACSA after a bolus feed of expressed breast milk (EBM) or EBM with added fortifier.

first. The examiner (RJM) was blinded to feed composition until analysis of data was complete. The median (range) feed volume was 20 (13-33) $\mathrm{ml} / \mathrm{kg}$. The median (range) duration of feed was 7 (5-11) minutes.

Gastric emptying was assessed by ultrasonically measuring the change in the crosssectional area of the gastric antrum that occurred following a feed, using the method that we have previously described in this journal. ${ }^{14}$ In brief, to measure the antral cross-sectional area (ACSA), real time ultrasound images of the same longitudinal section of the gastric antrum were obtained with the infant lying in the right lateral position. The vertebral bodies and the aorta, at the level of the superior mesenteric artery, were used as constant anatomical landmarks to ensure that the same longitudinal section of the gastric antrum was measured. Ultrasound images were obtained using the Hewlett Packard Ultrasound Imaging System (model HP Sonos 100) with a 7.5 MHz sector scanning probe (model $21415 \mathrm{~A}$ ). Images were frozen on the screen and the circumference of the gastric antrum marked with a steerable cursor. Using software available on the machine, the ACSA was then calculated.

Before each feed in the study, the stomach was aspirated. ACSA was measured before the feed, when the feed was completed, and then at 10 minute intervals until 30 minutes had elapsed. Measurement then continued at 15 minute intervals until two hours had elapsed or the pre-feed value was reached. Fifty per cent $\triangle$ ACSA was defined as the time taken for the ACSA to decrease by half of the maximum change seen during gastric filling. This value provided a simple single measurement of gastric emptying allowing subsequent comparison between feeds.

Informed parental consent was obtained before each case was studied and the protocol was approved by the local research ethics committee. Statistical analysis was performed using Student's paired $t$ test.

\section{Results}

One set of paired studies was successfully carried out on all infants. All seemed to tolerate the procedure well, with no infant vomiting or seeming to suffer undue distress. The mean (standard error) $50 \% \triangle \mathrm{ACSA}$ for EBM alone and for EBM with added fortifier was $46(5 \cdot 5)$ and $47 \cdot 2(5 \cdot 4)$ minutes, respectively. The mean difference (standard error) in $50 \%$ $\triangle$ ACSA between the two feeds was $1.48(4.9)$ minutes. This difference was not significant. The patterns of gastric emptying following both feeds were also virtually the same as shown in fig 1 .

There was considerable variation in some infants between the $50 \% \triangle$ ACSA of the two feed types (fig 2). There was also variation in the $50 \% \triangle A C S A$ among individual infants for each feed (fig 2), but there was no discernible trend. This variation did not seem to be related to volume of feed, gestation, or postnatal age.

\section{Discussion}

A study was performed to compare the gastric emptying in low birthweight infants with EBM alone with EBM with added fortifier, by assessing the change in ACSA measured by real time ultrasound.

In two telephone surveys, one in 1987 and one in 1994, of all regional neonatal units in the United Kingdom (unpublished data), we found a dramatic increase in the practice of fortifying breast milk. This increase was from $5 \%$ of units in 1987 to $85 \%$ in 1994 . The surveys also revealed that virtually all of this increase involved the use of specifically designed, commercially available fortifiers. The results of this study are therefore relevant to almost all neonatal units.

The gold standard for estimation of gastric emptying involves radioisotope studies but this requires irradiation and the use of a gamma camera. This method is not suitable for the assessment of low birthweight infants. Marker dilution techniques have been used in preterm infants, ${ }^{1516}$ but none is entirely satisfactory. Applied potential tomography (APT), a form of electric impedance tomography, has been used in term infants, ${ }^{17}$ is currently being

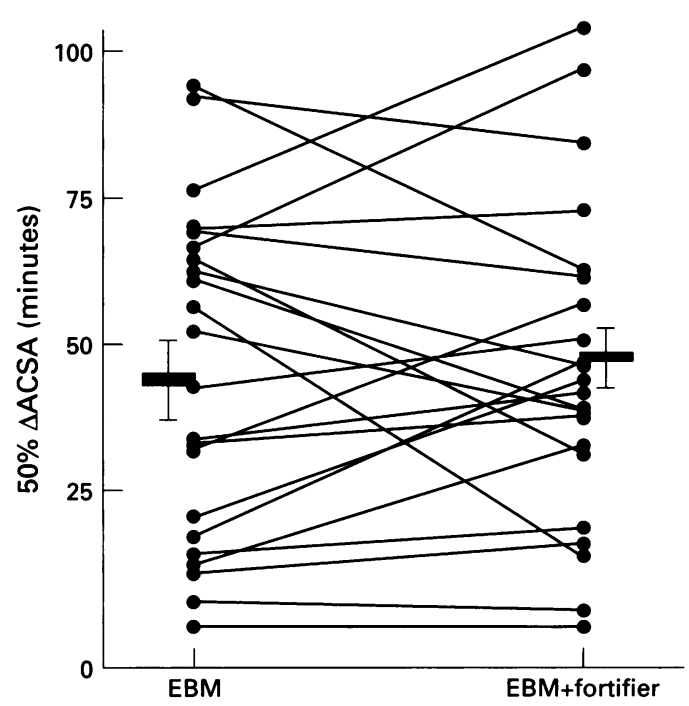

Figure 2 Half gastric emptying times ( $50 \% \triangle A C S A)$ for expressed breast milk (EBM) and EBM with added fortifier within individual infants $(n=22)$. Mean (SE) value for each feed is shown by a broad horizontal line. 
explored in low birthweight infants. The ultrasonic method used in this study has been validated and used to study gastric emptying in low birthweight infants. ${ }^{12} 14$

The 50\% $\triangle$ ACSA for EBM alone has been measured as 35 minutes by Newell et al ${ }^{14}$ and 36 minutes by Ewer et al, compared with 72 minutes for formula milk. ${ }^{12}$ The longer gastric emptying times for EBM seen in this study may reflect the study population. In the present study the median gestation and birthweight was lower, and a larger proportion of infants were small for gestational age (55\%). The differences in the $50 \% \triangle \mathrm{ACSA}$ between the two feeds, and the difference among individual infants for each feed, probably reflects the natural physiological variability of gastric emptying, ${ }^{12}$ but warrants further exploration. Our study was not designed to detect any relation between gastric emptying and volume of feed, gestation, or postnatal age. This precludes any conclusion being made from our observation that the variation in gastric emptying was not related to these variables.

There are currently three commercially available fortifiers in the United Kingdom: Breast Milk Fortifier (Cow \& Gate), Eoprotin (Milupa), and Enafamil (Mead Johnson). Fortifying breast milk has been shown to have a beneficial effect on weight gain and linear growth in low birthweight infants. ${ }^{7-9}$ Breast Milk Fortifier, which was the fortifier used in this study, raises the nutritional content of every $100 \mathrm{ml}$ of EBM by $10 \mathrm{kcal}$ energy, $0.7 \mathrm{~g}$ protein, and $2 \mathrm{~g}$ carbohydrate, as well as providing numerous extra vitamins and minerals. Eoprotin and Enafamil are virtually identical to Breast Milk Fortifier, the only major difference being that Eoprotin contains a very small amount of fat. On the basis of their similarity it is unlikely that either Eoprotin or Enafamil would have any noticeably different affect on gastric emptying.

Inadequate gastric emptying is associated with poor tolerance of milk feeds, a common problem in low birthweight infants. This is particularly so when enteral feeding is first introduced. There was good reason to hypothesise that the addition of fortifier to breast milk would retard gastric emptying. ${ }^{11-13}$ These data show the absence of any such effect following the addition of fortifier to EBM. The quantity or composition of fortifier may be insufficient to produce an effect. An alternative explanation is that EBM has a prokinetic property, actively promoting gastric motility.

We conclude, therefore, that in the low birthweight infant who may benefit from the fortification of breast milk, this form of nutritional supplementation may be used without retardation of gastric emptying. By implication, therefore, this form of supplementation will have no adverse affect on the tolerance of breast milk.

Dr Robert McClure is currently a Neonatal research fellow and is generously supported by the Nutricia Research Foundation. We are indebted to the nursing staff of the regional neonatal intensive care unit at St James's University Hospital, Leeds, for
their help and cooperation, to Dr P R F Dear for advice and support, and to Miss $M$ Jones for assistance in the preparation of the manuscript.

1 Standing Committee on Nutrition of the British Paediatric Association. Is breast feeding beneficial in the UK? Arch Dis Child 1994; 71: F376-80.

2 Harzer G, Haschke E. Micronutrients in human milk. In: Renner E, ed. Micronutrients in milk and milk-based food products. London: Elsevier, 1989: 125-237.

3 Roberts AK, van Biervliet JP, Harzer G. Factors of human milk influencing the bacterial flora of infant faeces. In: Schaub J, ed. Composition and physiological properties of human milk. London: Elsevier, 1985: 259-69.

4 Atkinson SA, Radde IC, Anderson GH. Macromineral balances in premature infants fed their own mothers' milk or formula. 7 Pediatr 1983; 102: 99-106.

5 Putet G, Senterre J, Rigo J, Salle B. Nutrient balance, energy utilization, and composition of weight gain in very ow birthweight infants fed pooled human milk or a preterm formula. $\mathcal{F}$ Pediatr 1984 ; 105: 79-85.

6 Rönholm KAR, Sipila I, Slimes MA. Human milk protein supplementation for the prevention of hypoproteinaemia without metabolic imbalance in breast milk-fed, very low birthweight infants. $\mathcal{F}$ Pediatr 1982; 101: 243.

7 Modalou HD, Lim MO, Hansen JW, Sickles V. Growth, biochemical status, and mineral metabolism in very low irthweight infants receiving fortified preten milk. F Pediatr Gastroenterol Nutr 1986; 5: 762-7.

8 Carey DE, Rowe JC, Goetz CA, et al. Growth and phosphorus metabolism in premature infants fed human milk, phorus metabolism in premature infants fed human milk, Dis Child 1987; 141: 511-15.

9 Greer FR, McCormick A. Improved bone mineralization and growth in premature infants fed fortified own mothers' milk. F Pediatr 1988; 112: 961-9.

10 ESPGAN. Nutrition and feeding of preterm infants. Acta Paediatr Scand 1987; 336: 1-14.

11 Siegel M, Lebenthal E, Krantz B. Effect of caloric density on gastric emptying in premature infants. 7 Pediatr 1984; 104: 118-22.

12 Ewer AK, Durbin GM, Morgan MEI, Booth IW. Gastric emptying in preterm infants. Arch Dis Child 1994; 71: F24-7.

13 Cavell B. Gastric emptying in preterm infants. Acta Paediatr Scand 1979; 68: 725-30.

14 Newell SJ, Chapman S, Booth IW. Ultrasonic assessment of gastric emptying in the preterm infant. Arch Dis Child 1993; 69: F32-6.

15 Husband J, Husband P. Gastric emptying of water and glucose solutions in the newborn. Lancet 1969; ii: 409-11.

16 George JD. New clinical method for measuring the rate of gastric emptying: the double sampling test meal. Gut 1968; 9: 237-42

17 Nour S, Mangnall YF, Dickson JAS, Pearse R, Johnson AG. Measurement of gastric emptying in infants with pyloric stenosis using applied potential tomography. Arch Dis Child 1993; 68: 484-6. 